Canadian Journal of Experimental Psychology 54(4):266-273, 2000.

\title{
Awareness during Drowsiness: Dynamics and Electrophysiological Correlates
}

\author{
Scott Makeig* \\ Naval Health Research Center \\ San Diego CA \\ Tzyy-Ping Jung \\ Institute for Neural Computation \\ University of California, San Diego \\ Terrence J. Sejnowski \\ Howard Hughes Medical Institute \\ The Salk Institute for Biological Studies \\ La Jolla CA
}

*Current address:

Scott Makeig, Ph.D.

The Salk Institute

10010 N. Torrey Pines Rd.

La Jolla CA 92037

USA

Tel: (858) 453-4100x1455

Fax: (858) 587-0417

E-mail: scott@salk.edu 


\section{Awareness during Drowsiness: Dynamics and Electrophysiological Correlates}

Summary: During drowsy periods, performance on tasks requiring continuous attention becomes intermittent. Previously, we have reported that during drowsy periods of intermittent performance 7 of 10 participants performing an auditory detection task exhibited episodes of non-responding lasting about $18 \mathrm{~s}$ (Makeig and Jung, 1996). Further, the time patterns of these episodes were repeated precisely in subsequent sessions. The $18 \mathrm{~s}$ cycles were accompanied by counterbalanced power changes within two frequency bands in the vertex EEG (near $4 \mathrm{~Hz}$ and circa $40 \mathrm{~Hz}$ ). In the present experiment, performance patterns and concurrent EEG spectra were examined in four participants performing a continuous visuomotor compensatory tracking task in 15-20 minute bouts during a 42-hour sleep deprivation study. During periods of good performance, participants made compensatory trackball movements about twice per second attempting to keep a target disk near a central ring. Autocorrelations of time series representing the distance of the target disk from the ring center showed that during periods of poor performance, marked near $18 \mathrm{~s}$ cycles in performance again appeared, with phases of poor or absent performance accompanied by an increase in EEG power that was largest at 3-4 Hz. These studies show that in drowsy humans, opening and closing of the gates of behavioral awareness is marked not by the appearance of $(12-14 \mathrm{~Hz})$ sleep spindles, but by prominent EEG amplitude changes in the low theta band. Further, both EEG and behavioral changes during drowsiness often exhibit stereotyped $18 \mathrm{~s}$ cycles.

\section{Introduction}

During drowsiness, both the participant's performance and their awareness of the external environment become intermittent. There has been relatively little study of the dynamics of this intermittency. An early report on behavioral experiments requiring continuous performance reported that participants seem disposed to make errors at intervals of about 20 seconds, but attempts to find reliable periodicities in performance have not produced strongly consistent results (Augenstein, 1955). The human electroencephalogram (EEG), often recorded in sleep studies, is traditionally thought of as comprising a series of higher and lower frequency bands whose strengths differ at differing levels of arousal and consciousness (reviewed in Bastien, Ladouceur and Campbell, this issue). Higher frequencies usually accompany arousal, wakefulness and focused attention. Thus, the EEG of an awake, alert and highly attentive person might contain oscillations at beta frequencies $(15-25 \mathrm{~Hz})$, while the same person's EEG during deep relaxation might display prominent $10-12 \mathrm{~Hz}$ alpha activity. EEG frequencies above $25-30 \mathrm{~Hz}$ are often said to be in the gamma band, within which frequencies near $40 \mathrm{~Hz}$ have been of particular interest in studies of attention (Freeman, 1975; Bird, Newton, Sheer, and Ford, 1978; Galambos, Makeig, and Talmachoff, 1981; Gray and Singer, 1989). On the other hand, high amplitude activity at lower frequencies regularly accompanies sleepiness, sleep and unconsciousness (Makeig and Inlow, 1993; Ogilvie, Simons, Kuderian, et al., 1991; see also Colrain, Di Parsia, and Gora, this issue). During the transition from a waking to a sleeping state, the EEG typically loses its prominent alpha and beta frequencies as lower frequency (circa $4 \mathrm{~Hz}$ ) theta activity appears (Makeig and Jung 1995). When the sleeper enters deeper sleep (or coma) and becomes unconscious of the normal external environment, large amplitude $0.5-2 \mathrm{~Hz}$ delta waves may be observed. There are exceptions to the rule hat higher frequencies occur during higher levels of arousal. Most notably, $2-14 \mathrm{~Hz}$ rhythmic waveforms known as sleep spindles occur during early sleep. 
The neural dynamics of sleep transition have also often been investigated in animals. Steriade and colleagues have reported that $4-12 \mathrm{~Hz}$ thalamocortical spindles triggered in the reticular nucleus of the thalamus in cats reduce or suppress the receptivity of cortex to afferent stimulation, thereby reducing sensory awareness during the transition to sleep (Steriade, 1994). It would appear that in order to sleep, sensory input must be inhibited early in the processing pathway. Although frequency of these cat 'sleep spindles' is somewhat lower than the 12-14 Hz human sleep spindles that are the hallmark of the transition to (Stage 2) sleep, it is tempting to assume that human sleep spindles have a similar function. Indeed, Cote, Epps, and Campbell (2000) indicate that human auditory evoked potentials show signs of sensory inhibition when stimuli are presented slightly before the occurrence of a spindle.

However, not all frequencies within the EEG are correlated with changes in human performance during drowsiness. Makeig and Jung (1996) studied operators who were engaged in a simulated passive sonar auditory detection task. They examined the EEG spectra surrounding 'lapses' (targets eliciting no response) and 'hits' (targets eliciting responses) during drowsy periods (defined as containing both hits and lapses within $30 \mathrm{~s}$ of the event). EEG amplitude changes predicted the behavioral response in only two frequency ranges. Immediately before lapses, power (mean square amplitude) between $2-5 \mathrm{~Hz}$ increased and power near $40 \mathrm{~Hz}$ decreased. Prior to hits, the opposite pattern was observed. We here label the $2-5 \mathrm{~Hz}$ activity observed during sleep onset and behavioral intermittency as low theta.

These drowsiness-related perturbations in the EEG spectrum began, on average, $9 \mathrm{~s}$ before stimulus presentation and returned to baseline $9 \mathrm{~s}$ after it (Makeig and Jung, 1996). Both the theta and gamma band power changes showed the same 18 s cycle. Moreover, detailed examination of the time course of performance in these episodes (computed as the relative probability of making a hit or lapse immediately before or after a given hit or lapse target) showed the same $18 \mathrm{~s}$ cycle. During drowsy periods, $9 \mathrm{~s}$ before a hit (or lapse) target the probability that a participant would respond to a preceding target began to rise (or fall). About $9 \mathrm{~s}$ after a hit (or lapse) target, participant response probability returned to baseline. This response probability trajectory was mirrored by parallel changes in low theta EEG power. The overall mean circa $18 \mathrm{~s}$ cycle was seen in the behavioral response patterns of 7 of the 10 participants, and tended to occur within longer 'waves' of drowsiness lasting two minutes or more. Finally, the performance and theta power trends for each of the participants were precisely replicated in second task sessions recorded some days later.

The observed pattern of counterbalanced EEG changes near $4 \mathrm{~Hz}$ and near $40 \mathrm{~Hz}$ accompanying transient losses of awareness generally fit with many reports correlating $40 \mathrm{~Hz}$ EEG power increases with alert, focused attention and consciousness (Freeman, 1975; Bird, Newton, Sheer, and Ford, 1978; Rogeul, Bouyer, Dedet, and Debray., 1979; Gross and Gottman, 1999) and irregular bursts of circa $4 \mathrm{~Hz}$ theta-band EEG with drowsiness, hypnogogy and the loss of consciousness (Williams, Granda, Jones, Lubin, and Armington, 1962; Schacter, 1977).

In the same experiments, changes in EEG power within the broad (6-35 Hz) intermediate frequency range between low theta and gamma did not predict changes in responsiveness during drowsiness. Instead, in accord with a previous report, power in these other frequencies was perturbed briefly about $1 \mathrm{~s}$ after stimulus onset (Makeig, 1993). However, during drowsiness the sign of this event-related spectral perturbation (power increase or decrease) varied according to the behavioral response (hit or lapse) (Makeig and Jung, 1996). The intermediate (6-35 Hz) frequency range included the 12-14 Hz range associated with human sleep spindles. Examination of mean spectral shifts associated with changes in response probability, averaged on the minute scale, showed a specific increase in power in the sleep spindle range only during extended periods of 
unresponsiveness (possibly signaling transition to Stage II sleep) and not during earlier periods of intermittent responding.

Here, we report similar $18 \mathrm{~s}$ performance cycles during periods of intermittent performance in a very different type of continuous performance task, a visuospatial compensatory tracking task requiring frequent $(\sim 2 / s)$ trackball movements. Data for this study drawn from concurrent EEG and performance data collected during a larger sleep deprivation study carried out by Dr. David Dinges and colleagues at the University of Pennsylvania (Dinges, Mallis, Maislin, and Powell, 1998). The purpose of the overall study was to compare proposed methods for monitoring subject alertness during sleep deprivation. In the tracking task reported here, the dominant EEG spectral correlate of performance degradation was again a power increase at 3-4 Hz. We discuss our results in light of possible neurophysiological mechanisms.

\section{Methods}

\section{Participants}

Four young adults ( $\mathrm{x}$ men, $\mathrm{x}$ women) between the ages of $\mathrm{xx}$ and $\mathrm{xx}(\mathrm{Mean}=, \mathrm{SD}=)$ volunteered to participate in this experiment.

\section{EEG Recording}

The EEG was recorded from electrodes placed at left frontal (F3), right central (C4), right parietal (P4) and left occipital (O1) sites. The reference was the left mastoid (M1). Eye movements and blinks were monitored via two encephalographic (EOG) channels. The EEG and EOG activity were digitized at a sampling rate of $125 \mathrm{~Hz}$.

EEG spectra were extracted by Hanning-windowed fast Fourier analysis (FFT) performed on overlapping $2 \mathrm{~s}$ epochs from the continuous EEG data record. Epochs contaminated by EOG and muscle artifact were rejected. Data from the right-central EEG channel (C4) are reported here.

\section{Procedure}

Participants arrived in the laboratory in the early morning (10:00). They were then sleep deprived for 42 hours. During the 42 hour sleep deprivation period, participants performed 9-10 bouts of two tasks at roughly 4 hour intervals: a psychomotor vigilance task, designed by Dinges and Powell (1985) lasting $20 \mathrm{~min}$, followed after a 1-2 minute break by a continuous visuospatial compensatory tracking task (CTT) lasting $20 \mathrm{~min}$. (At one participant's request, CTT bouts were limited to 15 minutes). Staff technicians were instructed not to alert participants who stopped responding during either task. In the present study data from the CTT task are reported.

In the CTT, participants manipulate a trackball to counter unseen quasi-random forces tending to "blow" a circular disk off of an invisible slippery slope around the screen center marked by a visible ring. Participants attempt to use rapid, small trackball movements to maintain the disk near the center of the ring. At each program step (about 14 times per s), the disk is moved according to the vectorial sum of forces acting on it -- the unseen "wind" plus the simulated "force of gravity" plus a directed force proportional to the most recent trackball velocity. The CTT performance measure we employed was the average distance of the moving disk from the fixed central ring. (For more details and program code, see Makeig and Jolley (1996), available from http://www.cnl.salk.edu/ scott).

In constructing the moving-mean performance measure, the disk-distance time series was first rescaled from units of screen pixels to disk diameters, and then passed through an sigmoidal 
$\operatorname{erf}($ ) function whose lower asymptote was tied to the participant's best performance in the indicated baseline bout. Here, best performance was quantified as the 10th percentile in the participant's histogram of mean disk-distance smoothed using a 2-min window moved through the data in $2 \mathrm{~s}$ steps. In previous studies, changes in mean CTT disk distance have been associated with fatigue (Makeig and Jolley, 1996) and with concurrent changes in eye activity and performance consistent with drowsiness (Van Orden, Jung, and Makeig, 2000).

The disk distance measure was then linearly rescaled to [0,1] with the upper bound scaled to place its upper asymptote at the measured mean disk distance in the absence of any participant input (9.4 disk radii). The disk distance time series were then smoothed using a $90 \mathrm{~s}$ square window moved through the data in $1 \mathrm{~s}$ steps, producing an individual mean error measure that varied smoothly between 0 (best participant performance) and 1 (no participant input).

To look for periodicities in participant performance, the raw target-distance time series for each overlapping $90 \mathrm{~s}$ epoch used in computing the smoothed distance measure was autocorrelated and the resulting autocorrelation time series were sorted on mean disk distance in the same epochs. Autocorrelation refers to variably lagged correlations of a time series with itself. Here, for convenience, unscaled autocorrelations were computed using the MATLAB function, xcorr() (The MathWorks, Natick MA). Finally, the autocorrelation time series were converted to power spectra by FFT analysis. 


\section{Results}

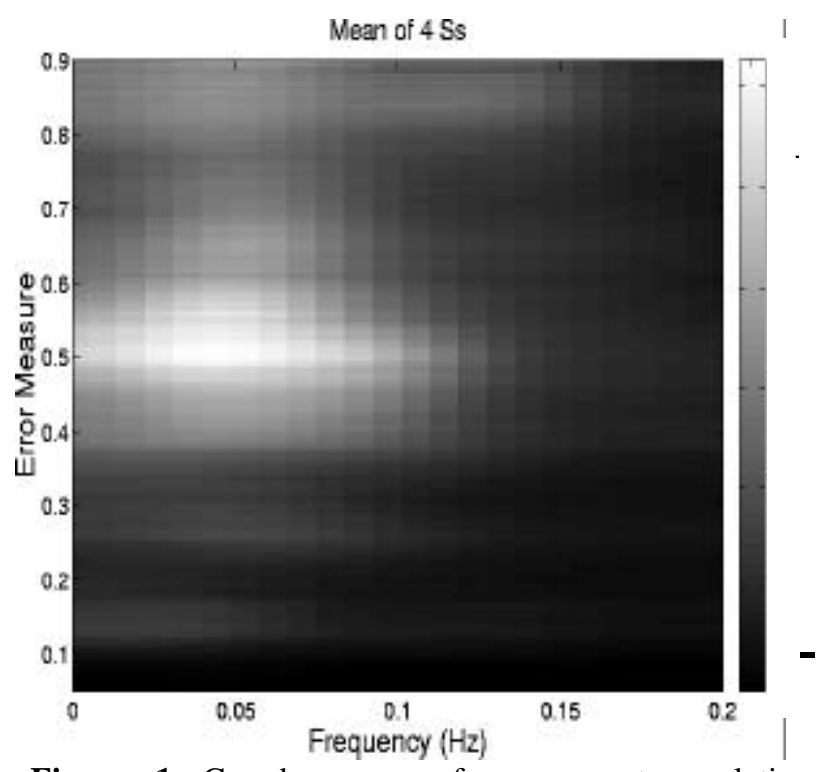

Figure 1. Grand mean performance autocorrelation (self-correlation of the raw disk-distance time series as a function of temporal lag (x-axis) and mean normalized disk distance (y-axis). Small normalized disk distances (0.30) indicate periods of relatively continuous good performance, while large distances (0.80) indicate periods of largely ineffective and/or absent performance. Data averaged over all four participants. Grey scale: lagged autocorrelation of the performance time series (relative units). Note the 15-20 s performance cycles during periods of relatively large disk distance (e.g., between 0.60 and 0.70 ), as indicated by the flanking light and dark spots near \pm 10 s and \pm 20 s lags respectively.

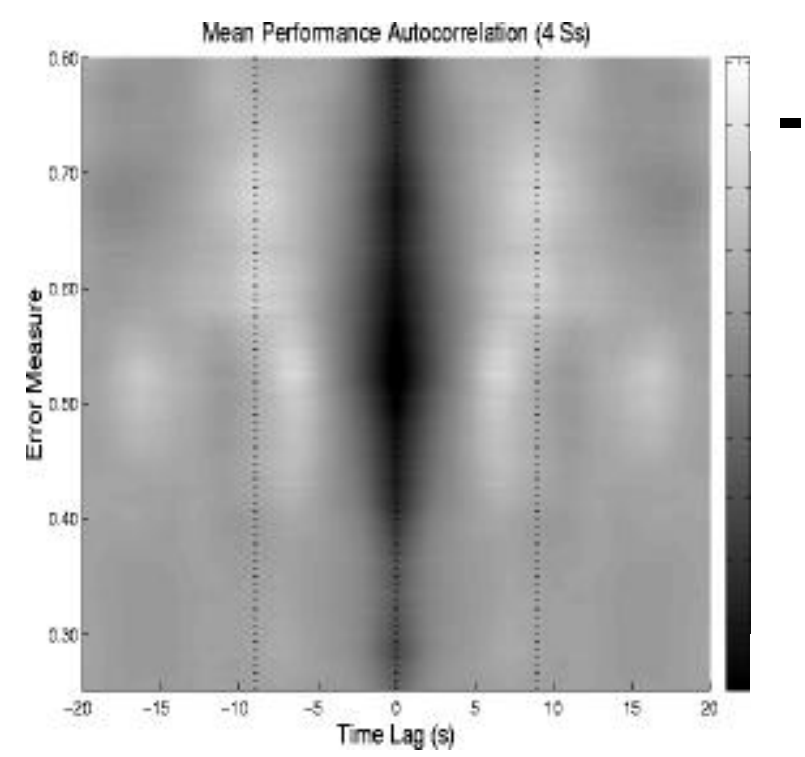

Figure 2. Grand mean power spectra (all 4 participants) of the performance time series autocorrelations (Fig. 1) sorted by performance error (normalized disk distance). $\mathrm{X}$-axis: cycle frequency $(\mathrm{Hz})$. Yaxis: Normalized disk distance. Gray scale: Mean cycle amplitude (change in disk distance per second). At relatively poor mean performance levels $(0.5$ to 0.7$)$, a peak in the performance autocorrelation spectrum appears near $0.05 \mathrm{~Hz}$, indicating the appearance of circa $20 \mathrm{~s}$ cycles between good (alert) and poor (or absent) performance (cf. Fig. 4 for example).

Figure 1 illustrates the mean performance autocorrelation time series sorted by smoothed disk distance and averaged across the four participants. An indication of a near $20 \mathrm{~s}$ performance cycle appeared at disk distances between 0.60 and 0.70 as a cycling between negative (light) and positive (dark) values at near $9 \mathrm{~s}$ intervals on either side of the central zero lag. Spectra of these same autocorrelation time series are illustrated in Figure 2, again sorted by disk distance. The near $18 \mathrm{~s}$ cycle length was confirmed by this analysis at normalized disk distances between 0.50 and 0.70 . This $18 \mathrm{~s}$ cycling was expressed exactly in the autocorrelation spectra of two of the participants, while for the other two participants the implied periodicity was weaker and somewhat longer. 


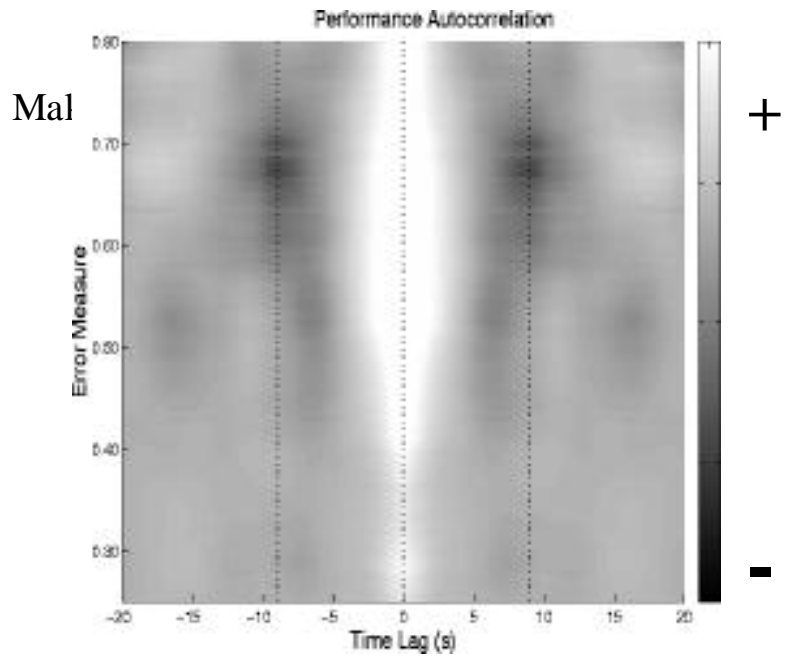

Figure 3. Autocorrelations of disk distance time series for one participant sorted by normalized disk distance. Gray scale: unnormalized autocorrelation in relative units (here dark is positive). At disk distances near 0.70, a marked circa $18 \mathrm{~s}$ lag correlation appears.
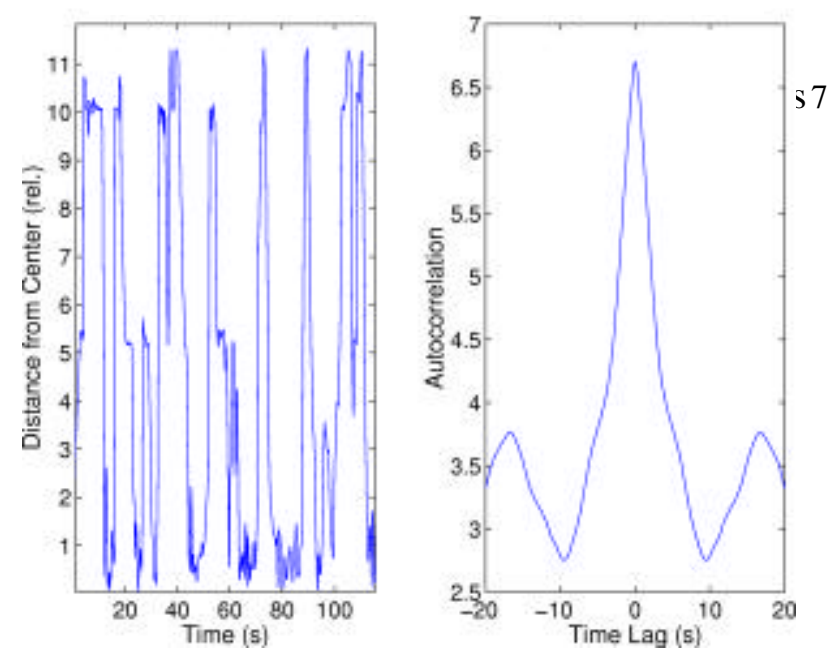

Figure 4. (left panel) Time course of performance fluctuations (actual disk distances in disk radii) at the beginning of a bout recorded after $32 \mathrm{hr}$ of sleep deprivation (same participant as Fig. 3), exhibiting marked circa 18 s performance cycles. (right panel) An unnormalized autocorrelation of the same performance time series shows positive (correlation) peaks at time lags near $\pm 18 \mathrm{~s}$, confirming the predominant $18 \mathrm{~s}$ performance periodicity in the record.

Figure 3 shows the sorted autocorrelations of the performance time series for one participant, averaged over 8 task bouts, showing the $18 \mathrm{~s}$ cycle at disk distances near 0.70 . A marked example of near $18 \mathrm{~s}$ cycling behavior from this participant is shown in Figure 4. The left panel plots the distance of the disk from the target ring during the first two minutes of a task bout performed after 32 hours of sleep deprivation. Periods of good performance (i.e. low disk distance, for example near $80 \mathrm{~s}$ ) alternate with periods in which the target escaped to levels near 1.0 compatible with complete lack of participant input. The autocorrelation of this performance time series (right panel) demonstrates the dominant $18 \mathrm{~s}$ periodicity.

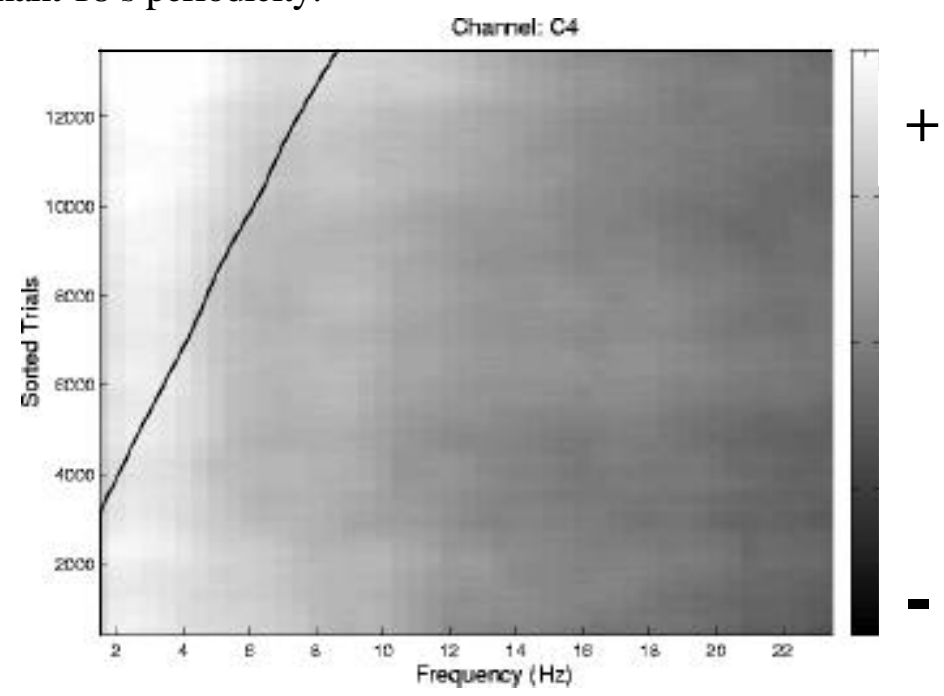

Figure 5. EEG spectra at right central site C4 in 13,000 $2 \mathrm{~s}$ task data windows (from all 4 participants) sorted by normalized disk distance in the $90 \mathrm{~s}$ window centered on the $2 \mathrm{~s}$ data window. X-axis: EEG frequency (Hz). Y-axis: sorted trial number. Gray scale: relative log EEG spectral power. The xvalues of points on the diagonal black trace indicate the normalized disk distance (times 10). Note the increase in EEG power between 2-5 Hz power during periods of relatively poor performance (e.g., normalized disk distances above 0.5 ). 
Figure 5 shows the grand mean EEG spectra for all four participants at right central electrode site $\mathrm{C} 4$, constructed by first sorting the spectra for each of the 13,000 $3 \mathrm{~s}$ trials by disk distance then smoothing the resulting image with a 0.05 -wide smoothing window. The prevailing disk distance is shown in the figure by a thick black line whose xvalue is ten times the prevailing disk distance (e.g., the thick line $\mathrm{x}$-value of 7 represents disk distance $=0.70)$. The figure shows that the general increase in low-frequency EEG power during poor performance (i.e., at relatively large disk distances) was largest near $3 \mathrm{~Hz}$. The circa $3-\mathrm{Hz}$ peak was present in data from all four participants. However, no specific increase at sleep spindling frequencies $(12-14 \mathrm{~Hz})$ is visible even at maximum disk distance.

\section{Discussion}

These results for a visuospatial task performed during sleep deprivation replicate our previous findings using an auditory detection task. In the previous study, participants had not been sleep deprived. Participants simply had to monitor a noise stream for brief amplitude shifts and respond to each shift with a button press. In the compensatory tracking task reported here, participants had to continuously observe and attempt to compensate for small changes in the rate and direction of continuous movement of a target disk. During good performance, participants in this task make about 2 corrective calibrated hand and finger movements per second, whereas only about 10 button presses per minute were required in the auditory task. The similarity of the changes in performance and EEG dynamics observed in the two tasks suggest they may be common to drowsy performance in any sensorimotor task. For both tasks, the most prominent change in the EEG spectrum correlated with performance degradation is an increase in power in the $2-5 \mathrm{~Hz}$ low theta range. This increase was found whether the data were averaged over seconds or minutes. For both tasks, an $18 \mathrm{~s}$ periodicity in performance and low theta EEG was observed during periods of intermittent performance during drowsiness.

Cross-correlation analysis on EEG spectra and performance time series were carried out for a single participant using consecutive $2 \mathrm{~s}$ windows. The resulting cross-correlation time series was then sorted by mean performance computed in a $90 \mathrm{~s}$ window centered on each epoch. Results confirmed the relation of $2-5 \mathrm{~Hz} \log$ theta EEG power to $18 \mathrm{~s}$ performance cycles at high disk distances (Fig. 5). The correlation between performance and gamma band EEG power was however very small. Possibly such a correlation may have been masked by EEG variability introduced by the constant random movements of the target disk, the difficulty of the task, and/or the frequent, rapid required finger movements.

Notably, the circa $18 \mathrm{~s}$ performance cycling seen in 7 of 10 participants for the auditory detection task (Makeig and Jung 1996) again occurred for at least 2 of the 4 participants in the compensatory tracking task. Thus, as was first reported from visual inspection of EEG tracings during sleep deprivation (Williams et al., 1962), the opening and closing of the "gate" of awareness and task performance capacity during drowsiness tends to occur in characteristic $18 \mathrm{~s}$ cycles, and is accompanied or indexed by amplitude changes in EEG activity in the $2-5 \mathrm{~Hz}$ low theta range rather than by the appearance of sleep spindles. Colrain et al. (this issue) also report that marked changes in both auditory and respiratory evoked potentials occur when the EEG is dominated by theta activity during stage 1 of sleep. It would be interesting to learn whether the 18 s cycles also appear as EEG fluctuations in participants who attempt to go to sleep, rather than in participants who (as here) attempt to stay awake. 
The $18 \mathrm{~s}$ cycling behavior is reminiscent of the alternating brief periods of relative alertness and drowsiness often seen in other mammals. Rajkowski and colleagues have presented evidence that changes in noradrenergic activation in the locus coeruleus precedes both the EEG spectral and accompanying behavioral vigilance shifts in monkeys (Rajkowski, Kubiak, and Aston-Jones, 1994). The locus coeruleus is one of a number of subcortical loci involved in modulating cortical activity and awareness. It is quite possible that their effects on cortical processing might be indexed by spectral shifts in the scalp EEG such as those reported here. In the present study, spectral estimation was applied to recordings from a single scalp electrode. Studies of these or similar data recorded at larger numbers of EEG electrodes using Independent Component Analysis (Makeig et al., 1996) might also reveal more information about the spatial sources of the EEG changes and their dynamics. Elsewhere, we have shown that the relationship between EEG spectral changes and performance impairments in drowsiness is sufficiently robust that individualized EEG models can accurately estimate performance in near real-time from EEG data (Makeig and Inlow, 1993; Jung, Makeig, Stensmo, and Sejnowski, 1997).

The results presented here reinforce four conclusions about dynamics of human awareness, concomitant performance changes, and EEG spectral power changes during drowsiness: (1) During periods of marked drowsiness, stereotyped cycles alternating between wake-like and sleep-like performance and in EEG power often occur. (2) During these cycles, phases of poor or absent performance are paralleled by increases in low-frequency (circa $4 \mathrm{~Hz}$ ) theta activity. (3) Often, these cycles have near periods near 18 seconds. (4) These performance and EEG cycles occur in the absence of any specific increase in EEG power at (12-14 Hz) human sleep spindle frequencies.

\section{Authors' Note}

This work was supported by U.S. DOT grant DTNH22-93-D-07007, by the Office of Naval Research, by NASA, by the Howard Hughes Medical Institute and by the Swartz Foundation. The authors thank David Dinges, Melissa Mallis and John Powell of the University of Pennsylvania for providing the data and assisting in its analysis. The views expressed in this article are those of the authors and do not reflect the official policy or position of the Department of the Navy, Department of Defense, or the U.S. Government. 


\section{References}

Augenstein, L. G. (1995). Evidences of periodicities in human task performance. In G. Quastler (Ed.) Information Theory in Psychology: Problems and Methods. (pp. 245-277). Glencoe, IL: The Free Press.

Bastien, C. H., Ladouceur, C., \& Campbell, K. B. (2000). EEG characteristics prior to and following the evoked K-Complex. Canadian Journal of Experimental Psychology (this issue).

Bird, B. L., Newton, F. A., Sheer, D. E., \& Ford, M. (1978). Behavioral and electroencephalographic correlates of 40-Hz EEG biofeedback training in humans. Biofeedback and Self Regulation,1,13-28.

Colrain, I.M., Di Parsia, P., \& Gora, J. (2000). The impact of pre-stimulus EEG frequency on auditory evoked potentials during sleep onset. Canadian Journal of Experimental Psychology (this issue)

Coull, J. T., Buchel, C., Friston, K. J., \& Frith, C. D. (1999). Noradrenergically mediated plasticity in a human attentional neuronal network. Neuroimage, 10, 705-15.

Cote, K. A., Epps,T. M., \& Campbell, K. B. (2000). The role of the spindle in human information processing of high intensity stimuli during sleep. Journal of Sleep Research, 9, 19-26.

Dinges, D. F., \& Powell, J. W. (1985). Microcomputer analyses of performance on a portable, simple visual RT task during sustained operations. Behavioral and Research Methods, Instruments and Computers, 17, 652-655.

Dinges, D. F., Mallis, M., Maislin, G., \& Powell, J. W. (1998). Evaluation of techniques for ocular measurement as an index of fatigue and the basis for alertness management. U.S. Department of Transportation, National Highway Traffic Safety Administration, Final Report \# DOT HS 808762.

Freeman, W. (1975). Mass Action in the Nervous System. New York: Academic Press.

Galambos, R., Makeig, S., \& Talmachoff, P. (1981). $40 \mathrm{~Hz}$ auditory potential recorded from the human scalp. Proceedings of the National Academy of Sciences, 78, 2643-2647.

Gray, C. M., \& Singer, W. (1989). Stimulus-specific neuronal oscillations in orientation columns of cat visual cortex. Proceedings of the National Academy of Sciences, 86, 1698-702. 
Gross, D. W., \& Gotman, J. (1999). Correlation of high-frequency oscillations with the sleep-wake cycle and cognitive activity in humans. Neuroscience, $94,1005-18$.

Jung, T-P., Makeig, S., Stensmo, M., \& Sejnowski, T. J. (1997). Estimating alertness from the EEG power spectrum. IEEE Transactions on Biomedical Engineering, 44, 60-69.

Makeig, S. (1993). Event-related dynamics of the EEG spectrum and effects of exposure to tones. Electroencephalography and Clinical Neurophysiology, 86, 283-293.

Makeig, S., Bell, A. J., Jung, T-P., \& Sejnowski, T. J. (1996). Independent component analysis of electroencephalographic data. In D. Touretzky, M. Mozer, M. Hasselmo (Eds.), Advances in Neural Information Processing Systems 8, (pp. 145-151). Cambridge, MA: MIT Press.

Makeig, S., \& Inlow, M. (1993). Lapses in alertness: coherence of fluctuations in performance and the EEG spectrum. Electroencephalography and Clinical Neurophysiology, 86, 23-35.

Makeig, S. \& Jung, T-P., (1995) Changes in alertness are a principal component of variance in the EEG spectrum. NeuroReport 7, 213-216.

Makeig, S., \& Jung, T-P. (1996). Tonic, phasic and transient EEG correlates of auditory awareness in drowsiness. Cognitive Brain Research, 4, 15-20.

Makeig, S., \& Jolley, M. (1996). COMPTRACK: A compensatory tracking task for monitoring alertness (Technical Document 96-3C). San Diego: Naval Health Research Center.

Ogilvie, R. D., Simons, I. A., Kuderian, R. H., MacDonald, T., \& Rustenburg, J. (1991). Behavioral, event-related potential, and EEG/FFT changes at sleep onset. Psychophysiology, 28, 54-64.

Rajkowski, J., Kubiak, P., \& Aston-Jones, G. (1994). Locus coeruleus activity in monkey: Phasic and tonic changes are associated with altered vigilance. Brain Research Bulletin , 35, 607-616.

Rogeul, A., Bouyer, J. J., Dedet, L., \& Debray, O. (1979). Fast somato-parietal rhythms during combined focal attention and immobility in baboon and squirrel monkey. Electroencephalography and Clinical Neurophysiology, 46, 310-19. 
Steriade, M. (1994). Sleep oscillations and their blockage by activating systems. Journal of Psychiatry and Neuroscience, 19, 54-58.

Rajkowski, J., Kubiak, P., \& Aston-Jones, G. (1994). Locus coeruleus activity in monkey: phasic and tonic changes are associated with altered vigilance. Brain Research Bulletin, 35, 607-16.

Rechtschaffen, A., \& Kales, A. (1968) A manual of standardised terminology, techniques, and scoring system for sleep stages of human subjects. Los Angeles: UCLA Brain Information Service.

Schacter, D. L. (1977). EEG theta waves and psychological phenomena: a review and analysis. Biological

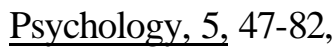

Van Orden, K., Jung, T-P., \& Makeig, S. (2000). Eye activity correlates of fatigue during a visual tracking task. Biological Psychology, 52, 221-40.

Williams, H. A., Granda, A., Jones, R., Lubin, A., \& Armington, J. (1962). EEG frequency and finger pulse volume as predictors of reaction time during sleep loss. Electroencephalography and Clinical Neurophysiology, 14, 64-70. 